\title{
Telemedicine and robotics: Paving the way to the globalization of surgery
}

\author{
S. Senapati ${ }^{a}$, A.P. Advincula ${ }^{\text {b,* }}$ \\ a Clinical Instructor and Fellow, Minimally Invasive Surgery, Department of Obstetrics and Gynecology, \\ University of Michigan Medical Center, Ann Arbor, MI USA \\ ${ }^{\mathrm{b}}$ Department of Obstetrics and Gynecology, University of Michigan Medical Center, USA
}

Received 25 August 2005; accepted 29 August 2005

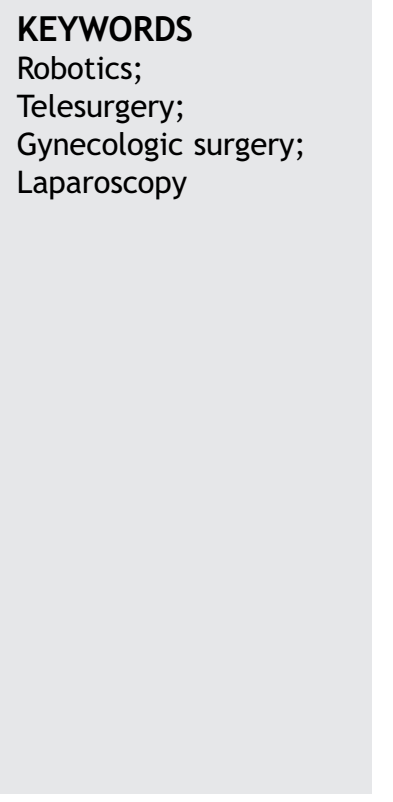

\begin{abstract}
The concept of delivering health services at a distance, or telemedicine is becoming an emerging tool for the field of surgery. For the surgical services, telepresence surgery through robotics is gradually being incorporated into health care practices. This article will provide a brief overview of the principles surrounding telemedicine and telepresence surgery as they specifically relate to robotics. Where limitations have been reached in laparoscopy, robotics has allowed further steps forward. The development of robotics in medicine has been a progression from passive to immersive technology. In gynecology, the utilization of robotics has evolved from the use of Aesop ${ }^{\circledR}$, a robotic arm for camera manipulation, to full robotic systems such as Zeus ${ }^{\circledR}$, and the daVinci ${ }^{\circledR}$ surgical system. These systems have not only been used directly for a variety of procedures but have also become a useful tool for conferencing and the mentoring of surgeons from afar. As this mode of technology becomes assimilated into the culture of surgery and medicine globally, caution must be taken to carefully navigate the economic, legal and ethical implications of telemedicine. Despite the challenges faced, telepresence surgery holds promise for more widespread applications.

(C) 2005 International Federation of Gynecology and Obstetrics. Published by Elsevier Ireland Ltd. All rights reserved.
\end{abstract}

\footnotetext{
* Corresponding author. Minimally Invasive Surgery Program and Fellowship, Department of Obstetrics and Gynecology, University of Michigan Medical Center, L 4000 Women's Hospital, 1500 East Medical Center Drive, Ann Arbor, MI 48109, USA. Tel:. +1 734764 8429; fax: +1 7346479727 .

E-mail address: aadvincu@umich.edu (A.P. Advincula).
}

\section{Introduction}

Telemedicine, literally meaning medicine from afar, "is the use of medical information exchanged from one site to another via electronic communications

0020-7292/\$ - see front matter (C) 2005 International Federation of Gynecology and Obstetrics. Published by Elsevier Ireland Ltd. All rights reserved. 
for the health and education of the patient or healthcare provider and for the purpose of improving patient care" [1]. One of the primary driving forces behind the original goal of telemedicine was enabling contemporary practices and resources to reach remote areas of the world where receiving and delivering adequate health care is a challenge [2]. The University of Nebraska was one of the first institutions in the United States to employ a means of supporting research and clinical efforts to remote areas of Nebraska in the 1950s. Other examples of early efforts in telemedicine include a link established between Massachusetts General Hospital and Logan Airport in Boston for emergency medical support. Unfortunately, these early ventures were very costly and politically heated. For years, few advances were made in telemedicine. It wasn't until 1986 that a satellite program was developed in the USA by the Mayo clinic between its main campus in Rochester, Minnesota and other clinics in Scottsdale, Arizona. A variety of applications have been realized for telemedicine since that time. Consultative services for second opinions, remote supervision for guidance in a technique, preoperative screening, follow-up visits, and videoconferencing for group meetings, formal lectures, and continuing medical education represent only a few of these applications.

\section{Telesurgery}

Telemedicine can be specifically applied to the surgical subspecialties as telesurgery. Telesurgery is defined broadly as the ability to perform surgery from a distance [3]. Telesurgery can take many forms, one of which is the ability to provide remote supervision for guidance in a technique. An example of this in obstetrics was the successful treatment of a pregnant woman in Santiago Chile using ultrasound and endoscopy directed by telesurgery from Tampa, Florida [4]. The patient was pregnant with monochorionic-diamniotic twins, one of which was an acardiac twin. Pregnancy termination was not available in Chile so after extensive counseling and approval from the ethics board of the Universidad de Chile, a successful amniorrhexis and photocoagulation of the umbilical cord of the acardiac twin was performed. In this case, an advanced telecommunication and teleconferencing system was able to allow the fetal surgery specialist in Tampa to guide the primary surgical team in Chile through the procedure. This particular application of telemedicine highlighted the ability to provide modern surgical skills to areas of need in remote locations.
As telemedicine and telesurgery have continued to make their presence known in cases such as the obstetric example, robotics appears to be playing an increasing role in their evolution. The use of robots in surgery has only come about within the past 25 years. The first application of a robot in surgery was in neurosurgery [5]. The original model, known as the PUMA 560, was used for neurosurgical stereotactic maneuvers under computed tomography (CT) guidance. In a similar fashion, a robotic system called the PROBOT was created to aide in transurethral resection of the prostate through guidance from a pre-operatively constructed three-dimensional image [6]. Soon, orthopedic surgery was utilizing a device called ROBODOC to aide in total hip replacements [7]. A common theme to these early designs was the way in which the robots functioned. They were developed to function autonomously with a preoperative plan or in a supervisory role. This passive role was soon going to evolve into a more active one with an immersive environment that became known as robotic telepresence technology.

The concept of robotic telepresence technology was born through the collaborative efforts of the Stanford Research Institute (SRI), the Department of Defense, and the National Aeronautics and Space Administration (NASA) [8]. The impetus for this concept was the need to be able to provide immediate operative care to wounded soldiers on the battlefield. Initial prototypes involved robotic arms that could be mounted on an armored vehicle in order to facilitate remote battlefield surgery. Soon thereafter, this technology became commercialized and robots were no longer just passive devices in surgery but ones that could be actively controlled in civilian operating rooms. Although robotic telepresence technology was initially created for cardiac surgery [9], it wasn't long before these developments were applied to the field of gynecology.

\section{Robotics in gynecology}

Technical advancements have clearly brought about improvements to modern day laparoscopy. These include high intensity xenon and halogen light sources as well as improved hand instrumentation. This technology has continued to grow by leaps and bounds in the area of minimally invasive gynecologic surgery. The days of a surgeon just peering through an eyepiece during a diagnostic laparoscopy have long passed thereby allowing more operative interventions to be performed. Studies have clearly shown that laparoscopic sur- 
gery allows faster recovery with improved cosmesis and less postoperative pain. Despite these technological advancements and benefits, more complex procedures such as the management of advanced endometriosis, and procedures that require extensive suturing such as myomectomy, pelvic reconstructive surgery, and tubal reanastomosis are typically still managed by laparotomy.

A great example of the slow adoption of minimally invasive surgical techniques to a gynecologic procedure has been hysterectomy. In 2002, Farquhar and Steiner reported that approximately $10 \%$ of hysterectomies were performed with the assistance of laparoscopy [10]. Approximately 600,000 hysterectomies are performed annually in the United States with the majority due to benign conditions [11,12]. Prior to the introduction of laparoscopic-assisted vaginal hysterectomy in the late 1980s, hysterectomies were approached by either a vaginal or abdominal route [13]. Althougha definite trend toward laparoscopic hysterectomy has been seen since the 1990s, hysterectomy via laparotomy remains the most common route.

One major obstacle to the more widespread acceptance and application of minimally invasive surgical techniques to gynecologic surgery has been the limitations encountered with conventional laparoscopy. These include counter-intuitive hand movement, two-dimensional visualization, and limited degrees of instrument motion within the body. In an attempt to overcome these obstacles, robotics has been looked upon as the solution.

One of the early predecessors and first applications of robotic technology to the field of gynecology was with a voice-activated robotic arm known as Aesop ${ }^{\circledR}$ (Computer Motion Inc. ${ }^{\circledR}$, Goleta, CA). The primary role of Aesop ${ }^{\circledR}$ was to operate the camera during laparoscopic surgery. One study by Mettler et al. compared the system to a surgical assistant holding the laparoscope during gynecologic surgery [14]. The authors found that the time required to perform surgery was faster with the robotic camera holder because it allowed the two surgeons to use both hands for operating thereby improving efficiency.

Another predecessor to the current platform of surgical robots was Zeus ${ }^{\circledR}$ (Computer Motion Inc. ${ }^{\circledR}$, Goleta, CA). Dr. Yulan Wang, one of the pioneering forces behind the design of the Zeus ${ }^{\circledR}$ robotic system outlined five essential features for a successful surgical robotic system: multiple robotic arms, improved ergonomics, enhanced dexterity of motion to eliminate intention tremors, higher magnification of the surgical field and finally, fast and reliable data transmission between the surgeon and his or her tools and imaging systems.
This end result was a system that was comprised of three remotely controlled robotic arms that were attached to the surgical table and a workstation called a robotic console. This console possessed the instrument controls while threedimensional vision was obtained with the aide of special glasses. The robotic arms operated the camera and provided the surgeon with two operating arms that possessed interchangeable "microwrist" instruments. Although these instruments more closely mimicked the movements of the human wrist when compared to conventional laparoscopic instruments, their movements were not totally instinctive.

Early studies reported on its successful application to tubal reanastomosis. In one prospective study, pregnancy rates were evaluated in ten patients with previous tubal ligations who underwent laparoscopic tubal reanastomosis using the identical technique used at laparotomy [15]. A post-operative tubal patency rate of $89 \%$ was demonstrated in 17 of the 19 tubes anastomosed with a pregnancy rate of $50 \%$ at one year. There were no complications or ectopic pregnancies.

The use of robotic technology to facilitate laparoscopic procedures in gynecology is clearly evident with these two early systems. The use of robotics in gynecology has only continued to increase over the past 5 years, particularly with the introduction of the latest FDA approved platform in surgical robotics, the daVinci ${ }^{\circledR}$ surgical system (Intuitive Surgical ${ }^{\circledR}$, Sunnyvale, CA). In fact, the daVinci ${ }^{\circledR}$ surgical system is the only actively produced robotic surgical system incorporating an immersive telepresence environment.

The daVinci ${ }^{\circledR}$ surgical system is comprised of three components [Fig. 1]. The first component is the surgeon console where the surgeon controls the robotic system remotely. A stereoscopic viewer as well as hand and foot controls is housed in this unit. The second component of the daVinci ${ }^{\circledR}$ surgical system is the InSite ${ }^{\circledR}$ vision system which provides the three dimensional imaging through a $12 \mathrm{~mm}$ endoscope. The third component of the daVinci ${ }^{\circledR}$ surgical system is the patient-side cart with telerobotic arms and Endowrist ${ }^{\circledR}$ instruments. Currently this system is available with either three or four robotic arms. One of the arms holds the laparoscope while the other two to three arms hold the various Endowrist ${ }^{\circledR}$. These laparoscopic surgical instruments are unique in that they possess seven degrees of freedom which replicates the full range of motion of the surgeon's hand. These seven degrees of freedom are 1) in and out movement, 2) axial rotation, 3) opening and closing of instrument, 4) lateral movement at the articulation, 5) 


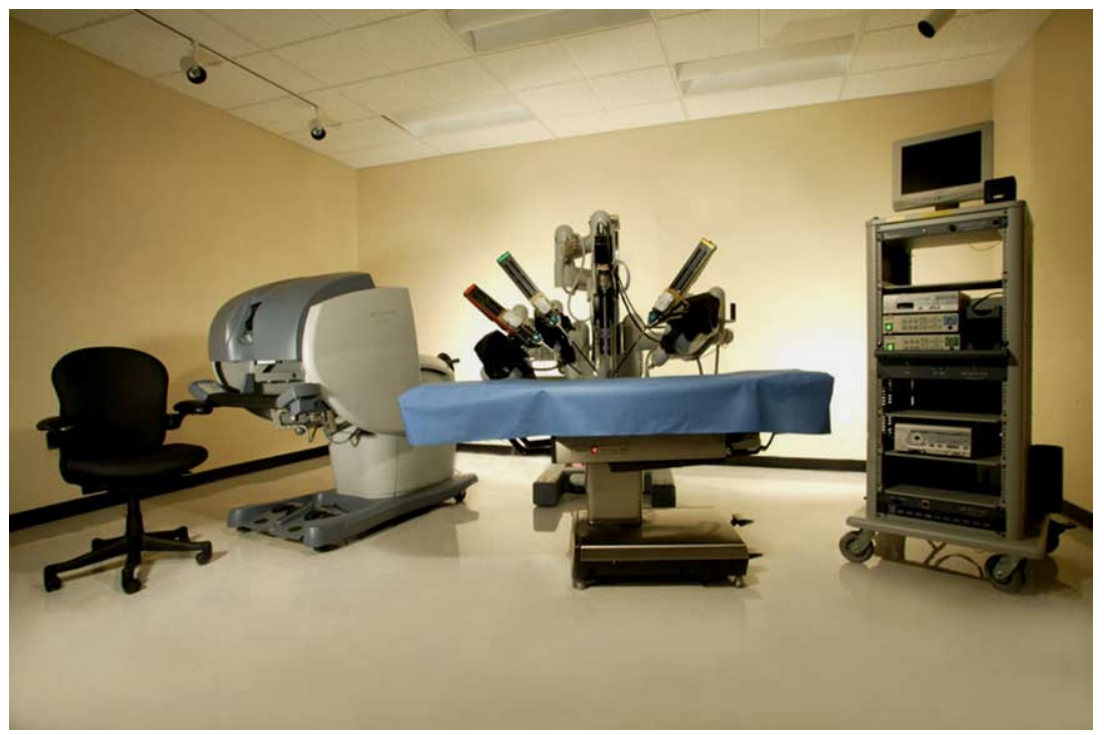

Figure 1 Photograph of the da Vinci ${ }^{\circledR}$ Robotic System. From left to right: surgeon's console, patient-side surgical cart, and InSite ${ }^{\circledR}$ vision tower. Photo courtesy of Intuitive Surgical ${ }^{\circledR}$, Inc.

vertical movement at the articulation, 6) right movement at each articulation, and 7) left movement at each articulation. A myriad of laparoscopic instruments are available such as needle drivers, Debakey forceps, and monopolar cautery spatulas. The significant improvement over earlier prototypes is that the seven degrees of freedom obtained at the surgeon console allow instrument manipulation to be instinctive. The fulcrum effect seen with conventional laparoscopy is eliminated.

In numerous studies across various disciplines, the daVinci ${ }^{\circledR}$ surgical system has been shown to be a safe and effective alternative to conventional laparoscopic surgery, particularly when dealing with complex pathology. In the area of gynecology, there are reports of robot-assisted laparoscopy for suture-based procedures where improved dexterity and precision coupled with advanced imaging is a huge benefit to the surgeon. The daVinci ${ }^{\circledR}$ surgical system has been applied to tubal reanastomosis, ovarian transposition, hysterectomy, myomectomy, and the repair of vaginal vault prolapse [16-20].

Although many of the limitations encountered with conventional laparoscopic instrumentation are overcome with robotics, additional challenges center on the training and acquisition of advanced skills. The surgeon's skill level and the lengthy training interval to attain laparoscopic competence have been known to affect the application of minimally invasive surgical techniques to procedures such as hysterectomy [21]. In an interesting report by Chapron et al. from Europe, training was found to be a major factor in the choice of technique when a review of the rate of laparoscopic hysterectomy in twenty-three French medical centers revealed that only nine centers carried out total laparoscopic hysterectomies [22].

When access to surgical training is available, the learning curve for conventional laparoscopy and prevention of associated complications are still significant limitations to widespread application. Although there are no absolute contraindications to many of the laparoscopic procedures in gynecology, a surgeon's experience and the pathology encountered remain the limiting factors for performing successfully. The use of robot-assisted laparoscopy may rapidly bridge the gap between assimilation of technique and the actual application of the procedure. A recent study by Sarle et al. confirmed this belief. The authors assessed the impact of robotics on surgical skills by comparing conventional laparoscopy with the daVinci ${ }^{\circledR}$ surgical system in the performance of four training drills. Surgeons completed drills faster with the robotic system. Most importantly, the study found that the playing field between novice and expert laparoscopic surgeons was leveled with the use of the robotic system [23].

\section{Telepresence surgery}

As discussed thus far, telesurgery by way of robotics has not only been shown to overcome many of the limitations seen with conventional laparoscopy and facilitate the acquisition of complex endoscopic skills but it is also defined broadly as the ability to perform surgery from a distance, otherwise known as telepresence surgery [3]. This was shown robotically in 2001. The distance barrier was broken in 
dramatic fashion when a New York to France telepresence surgery was completed. The physicians were able to operate with a time delay of less than $\mathbf{2 0 0}$ ms between the controls in New York and the action of the instruments on the patient in Strasbourg, France. Two communication systems were utilized to link both the video feed as well as a telephone link by a fiber optic service. The operation, entitled "Lindbergh" after the pilot who took the first transatlantic flight, was a successful cholecystectomy. It became a milestone in the history of telesurgery and inspired hope for those envisioning the continued globalization of surgery $[24,25]$.

One of the first telerobotic remote surgical services was designed in Canada. Anvari and colleagues created a program in 2003 by which the Zeus TS microjoint system (Computer Motion Inc. ${ }^{\circledR}$, Goleta, CA) was set up in Hamilton Ontario for the surgeon and the robotic arms were positioned on the patient in North Bay, Ontario-400 km away. They were able to utilize a pre-established commercial network as a communication link between the two locations. Twenty-one laparoscopic surgeries including fundoplications, sigmoid resections, hemicolectomies, an anterior resection and inguinal hernia repairs were completed successfully with this set-up. The two surgeons on either end were able to operate simultaneously with little time delay for communication and signal reception. They reported no significant complications [26].

\section{Telementoring}

Telementoring involves the remote guidance of a treatment or procedure where the caregiver has absent or limited experience with the featured technique [27]. It is an interactive method that facilitates a young surgeons' education by distant learning, tutoring of an expert surgeon, or having a remote surgeon acting as a preceptor to provide guidance through difficult operations [28]. Telementoring is supported by a videoconferencing system and it is an additional methodology to traditional didactics for clinicians and surgeons. It allows personal virtual training by computers and telecommunication systems.

One of the clear requirements of this process must be that a standard protocol be set for the global use of telementoring especially since the risk undertaken is much higher with this set-up. An important pre-requisite for the process is the skill level of the mentored individual in handling issues that may arise through the process. A fast reliable transfer of information is also required for constant, immediate communication with both audio and video feed. The visual feed should include views of both the outside of the patient as well as the internal operative field during laparoscopy. Robotic surgical systems may allow this process to take place. Telementoring through a robotic interface would provide an ability to train surgeons in new procedures and techniques from experts around the world. This in turn would improve the quality of training and allow the global dissemination of new surgical knowledge.

\section{Challenges}

Although robotic systems such as the daVinci surgical system provide advanced instrumentation and imaging, one limitation of the current state of the art in robot-assisted laparoscopy is the absence of tactile feedback to the surgeon operating the instruments remotely at the surgeon's console. The ability to allow tactile feedback to be relayed to the surgeon in robot-assisted laparoscopy has not yet occurred due to the expense of the technology. On the other hand, the improved visualization gained with the three-dimensional imaging seems to overcome this deficit for surgeons.

Costs may also be prohibitive as a robotic system such as the daVinci ${ }^{\circledR}$ surgical system retails for over $\$ 1,000,000$. Longer operative times in addition to disposable instruments and drapes also factor into the increased costs. Once robotics is applied to the telesurgical arena, costs increase exponentially and become one of the most common obstacles to establishing telesurgery services. The "Lindbergh" operation described earlier had a price tag of over one million euros [25]. Part of the initial overhead of this type of project is the communication system which represents one of the most critical components of a successful tele-operation system. The most common service used now is the asynchronous transfer mode (ATM) system, however; the limitation of using this system is the issue of access. Most hospitals currently do not have this system already set-up, therefore the initial costs may be more than what some institutions can afford.

Economics set aside and despite the great technological advances in robotic and digital communication, latency still remains an issue in long distance telesurgical ventures. The time delay between when the operator applies a certain motion and when the effect is seen on the patient's side has raised questions about the safety of 
telesurgery. Many surgeons have been able to overcome this obstacle; however, there is still a noticeable delay. Most surgeons have adopted 300 $\mathrm{ms}$ as the threshold above which the latency is not acceptable $[26,29]$. As this technology and means of communication reaches farther out and becomes more widespread, any decrease in latency will be an increase in the inherent safety of the procedure.

Ethical issues involving patient privacy and responsibility for the care of the patient are critical when dealing with a surgeon at the bedside and a surgeon operating from a remote location during a robotic telesurgery. Overall medical liability is an issue that is being addressed by those few already engaged in tele-operation efforts. For example in the "Lindbergh" operation, the surgery proceeded only after the French assumed all legal responsibility for the operation as both the on-site surgeons and patient were French. That particular surgery was performed uneventfully however, for future operations all parties need to have appropriate back-up plans for the unlikely situation that there is a systems failure with for example the surgical robot, or the communication lines.

Especially with telesurgery and telerobotics, full approval from the ethical/institutional review boards of both parties involved must be obtained prior to initiating the surgery. Responsibilities must be clearly delineated. Details such as medical privileges and reimbursement for the surgeons and insurance coverage for the patient should be completed prior to undertaking this type of endeavor. In one example, Anvari et al. established a formal legal agreement between not only the surgeons but also all hospital and equipment companies involved. This clearly specified each group's role and outlined each party's responsibilities [26].

\section{Conclusion}

Ideally, it is not hard to imagine an environment where physicians and patients are connected via a communication network that allows surgeons to collaborate from distant sites in order to provide cutting edge care to a patient with a rare disease in a remote part of the world. Not only would this be beneficial from both a consultative perspective and to the patient but also for the international exchange of ideas [30]. The role of robotics in the telesurgery movement presents the opportunity for the international exchange of new surgical techniques at a pace that is unprecedented. In doing so, credentialing and certification of surgeons may be accomplished while standardizing surgical training. Overall, evolving robotic and telesurgical technology may further improve patient outcomes while presenting new options on a global scale for the minimally invasive management of gynecologic pathology in the future. As a caution, care must be undertaken to evaluate not only the economic and ethical issues but also proper studies must be conducted in order to determine the exact role of robotic and telesurgical technology in the practice of minimally invasive gynecologic surgery.

\section{References}

[1] Rafiq A, Merrell RC. Telemedicine for access to quality care on medical practice and continuing medical education in a global arena. J Contin Educ Health Prof 2005;25:34- 42.

[2] Rosser JC, Gabriel N, Herman B, Murayama M. Telementoring and teleproctoring. World J Surg 2001;25:1438-48.

[3] Pande RU, Patle $Y$, et al. The telecommunication revolution in the medical field: present applications and future perspective. Curr Surg 2003;6:636-40.

[4] Quintero RA, Munoz H, Pommer R, Diaz C, et al. Operative fetoscopy via telesurgery. Ultrasound Obstet Gynecol 2002; 20:390- 1.

[5] Kwoh YS, Hou J, Jonckheere EA, Hayati S. A robot with improved absolute positioning accuracy for stereotactic brain surgery. IEEE Trans Biomed Eng 1988;35:153-60.

[6] Davies BL, Hibberd RD, Coptcoat MJ, Wickham JEA. A surgeon robot prostatectomy-a laboratory evaluation. J Med Eng Technol 1989;13:273-7.

[7] Bauer A, Borner M, Lahmer A. Clinical experience with a medical robotic system for total hip replacement. In: Nolte R, Ganz R, editors. Computer Assisted Orthopedic Surgery. : Hogrefe and HuberBern; 1999. p. 128-33.

[8] Satava RM. Robotic surgery: from past to future-a personal journey. Surg Clin North Am 2003;83:1-6.

[9] Diodato MD, Damiano RJ. Robotic cardiac surgery: overview. Surg Clin North Am 2003;83:1-12.

[10] Farquhar CM, Steinerm CA. Hysterectomy rates in the United States 1990-1997. Obstet Gynecol 2002;99:229-34.

[11] Wilcox LS, Koonin LM, Pokras R, Strauss LT, Xia Z, Peterson HB. Hysterectomy in the United States, 1988-1990. Obstet Gynecol 1994;83:549-55.

[12] Lepine LA, Hillis SD, Marchbanks PA, Koonin LM, Morrow B, Kieke BA, et al. Hysterectomy surveillance-United States, 1980-1993. MMWR CDC Surveill Summ 1997;46:1-15.

[13] Reich H, Decaprio J, McGlynn F. Laparoscopic hysterectomy. L Gynecol Surg 1989;5:213-6.

[14] Mettler L, Ibrahim $M$, Jonat W. One year of experience working with the aid of a robotic assistant (the voicecontrolled optic holder AESOP) in gynecologic endoscopic surgery. Hum Reprod 1998;13:2748-50.

[15] Falcone T, Goldberg JM, Margossian H, Stevens L. Robotically assisted laparoscopic microsurgical anastomosis: a human pilot study. Fertil Steril 2000;73:1040-2.

[16] Degueldre M, Vandromme J, Huong PT, Cadiere GB. Robotically-assisted laparoscopic microsurgical tubal reanastomosis: a feasibility study. Fertil Steril 2000;74: 1020 - 3.

[17] Molpus KL, Wedergren JS, Carlson MA. Robotically-assisted endoscopic ovarian transposition. JSLS 2003;7:59-62. 
[18] Diaz-Arrastia C, Jurnalov C, Gomez G, Townsend C. Laparoscopic hysterectomy using a computer-enhanced surgical robot. Surg Endosc 2002;16:1271- 3.

[19] Advincula AP, Song A, Burke W, Reynolds RK. Preliminary experience with robot-assisted laparoscopic myomectomy. J Am Assoc Gynecol Laparosc 2004;11(4):511- 8.

[20] Dimarco DS, Chow GK, Gettman MT, Elliott DS. Roboticassisted laparoscopic sacrocolpopexy for treatment of vaginal vault prolapse. Urology 2004;63:373-6.

[21] Wattiez A, Cohen SB, Selvaggi L. Laparoscopic hysterectomy. Curr Opin Obstet Gynecol 2002;14:417-22.

[22] Chapron C, Laforest L, Ansquer Y, Fauconnier A, Fernandez B, Breast $G$, et al. Hysterectomy techniques used for benign pathologies: results of a French multicenter study. Hum Reprod 1999;14:2464-70.

[23] Sarle R, Tewari A, Shrivastava A, Peabody J, Menon M. Surgical robotics and laparoscopic training drills. J Endourol 2004;18:63-7.

[24] Marecaux J, Leroy J, Gagner M. Transatlantic roboticassisted telesurgery. Nature 2001;413:379-80.
[25] Marescaux J, Leroy J, Rubino F, Smith M, Vix M, Simone M, et al. Transcontinental robot-assisted remote telesurgery: feasibility and potential applications. Ann Surg 2002; 235:487-92.

[26] Anvari M, McKinley C, Stein H. Establishment of the world's first telerobotic remote surgical suite. Ann Surg 2005; 241:460- 4

[27] Rosser JC, Gabriel N, Herman B, Murayama M. Telementoring and teleproctoring. World J Surg 2001;25:1438-48.

[28] Pande RU, Patle Y, Powers CJ, D'ancona G, Karamanoukian $\mathrm{H}$, et al. The Telecommunication Revolution in the Medical Field: Present Applications and Future Perspective. Current Surg 2003;6:636-40.

[29] Fabrizio MD, Lee BR, Chan DY, Stoianovici D, Jarrett TW, Yang C, et al. Effect of time delay on surgical performance during telesurgical manipulation. J Endourol 2000;14:133-8.

[30] Rafiq A, Merrell RC. Telemedicine for access to quality care on medical practice and continuing medical education in a global arena. J Contin Educ Health Prof 2005;25:34-42. 